\title{
Unlabeled $(2+2)$-free posets, ascent sequences and pattern avoiding permutations
}

\author{
Mireille Bousquet-Mélou1"; Anders Claesson" $\left.\right|^{1}$ : Mark Dukes ${ }^{3}$ \\ and Sergey Kitaev ${ }^{2}$ \\ ${ }^{1}$ CNRS, LaBRI, Université Bordeaux 1, 351 cours de la Libération, 33405 Talence, France. \\ ${ }^{2}$ The Mathematics Institute, Reykjavík University, 103 Reykjavík, Iceland. \\ ${ }^{3}$ Science Institute, University of Iceland, 107 Reykjavik, Iceland.
}

\begin{abstract}
We present statistic-preserving bijections between four classes of combinatorial objects. Two of them, the class of unlabeled $(\mathbf{2}+\mathbf{2})$-free posets and a certain class of chord diagrams (or involutions), already appeared in the literature, but were apparently not known to be equinumerous. The third one is a new class of pattern avoiding permutations, and the fourth one consists of certain integer sequences called ascent sequences.

We also determine the generating function of these classes of objects, thus recovering a non-D-finite series obtained by Zagier for chord diagrams. Finally, we characterize the ascent sequences that correspond to permutations avoiding the barred pattern $3 \overline{1} 52 \overline{4}$, and enumerate those permutations, thus settling a conjecture of Pudwell.

Résumé. Nous présentons des bijections, transportant de nombreuses statistiques, entre quatre classes d'objets. Deux d'entre elles, la classe des EPO (ensembles partiellement ordonnés) sans motif $(\mathbf{2}+\mathbf{2})$ et une certaine classe d'involutions, sont déjà apparues dans la littérature. La troisième est une classe de permutations à motifs exclus, et la quatrième une classe de suites que nous appelons suites à montées.

Nous déterminons ensuite la série génératrice de ces classes, retrouvant ainsi un résultat prouvé par Zagier pour les involutions sus-mentionnées. La série obtenue n'est pas D-finie. Apparemment, le fait qu'elle compte aussi les EPO sans motif $\mathbf{2}+\mathbf{2}$ est nouveau. Finalement, nous caractérisons les suites à montées qui correspondent aux permutations évitant le motif barré $3 \overline{1} 52 \overline{4}$ et énumérons ces permutations, ce qui démontre une conjecture de Pudwell.
\end{abstract}

Keywords: $(\mathbf{2}+\mathbf{2})$-free poset, interval order, pattern-avoidance, enumeration, ascent sequence, kernel method.

\footnotetext{
${ }^{\dagger}$ MBM was supported by the French "Agence Nationale de la Recherche", project SADA ANR-05-BLAN-0372.

¥AC and SK were supported by grant no. 060005013 from the Icelandic Research Fund.
} 


\section{Introduction}

This paper presents correspondences between four seemingly unrelated structures; unlabeled $(\mathbf{2}+\mathbf{2})$-free posets on $n$ elements, certain sequences of $n$ nonnegative integers called ascent sequences, a new class of permutations on $n$ letters, and finally certain involutions on $2 n$ points.

A poset is said to be $(\mathbf{2}+\mathbf{2})$-free if it does not contain an induced subposet that is isomorphic to $\mathbf{2}+\mathbf{2}$, the union of two disjoint 2-element chains. Fishburn [6] showed that a poset is $(\mathbf{2}+\mathbf{2})$-free precisely when it is isomorphic to an interval order. Another characterization is that a poset is $(\mathbf{2}+\mathbf{2})$-free if and only if the collection of strict principal down-sets can be linearly ordered by inclusion [5: 4].

Our ascent sequences have a simple recursive definition, given in Section 2 . We also define there the class of permutations we consider: they avoid a particular pattern of length three, but this type of pattern is new, in the sense that it does not admit an expression in terms of the dashed ${ }^{(i)}$ patterns introduced by Babson and Steingrímsson [1]. It is our hope that the results of this paper will stimulate research into these new patterns. We show how to deconstruct these permutations element by element, and how this gives a bijection with ascent sequences. In Section 3 we perform a similar task for $(\mathbf{2}+\mathbf{2})$-free posets.

In Section 4 we present a simple algorithm that given an ascent sequence $x$ computes what we call the modified ascent sequence, denoted $\widehat{x}$. Some of the properties of the permutation and the poset corresponding to $x$ are more easily read from $\widehat{x}$ than from $x$. We also explain how to go directly from a given poset to the corresponding permutation as opposed to via the ascent sequence. As an additional application, we show that the fixed points under $x \mapsto \widehat{x}$ are in one-to-one correspondence with permutations avoiding the barred pattern $3 \overline{1} 52 \overline{4}$. We count ascent sequences that are left unchanged by the map $x \mapsto \widehat{x}$, thus proving a conjecture of Lara Pudwell on the number of $3 \overline{1} 52 \overline{4}$-avoiding permutations.

In Section 5 we present statistics on the objects that are preserved under the stated bijections. In Section 6 we determine the generating function of ascent sequences (and thus, of $(2+2)$-free posets and pattern avoiding permutations), which turns out to be a rather complicated, non-D-finite series. This series has already been shown by Zagier [13] to count certain chord diagrams, or involutions, introduced by Stoimenow [12] to give upper bounds on the dimension of the space of Vassiliev's knot invariants of a given degree. In Section 7 we give a new proof of this result by establishing a bijection between these involutions and $(\mathbf{2}+\mathbf{2})$-free posets.

The proofs are omitted in this abstract, but can be found in the full version of the paper [2].

\section{Ascent sequences and pattern avoiding permutations}

Let $\left(x_{1}, \ldots, x_{i}\right)$ be an integer sequence. The number of ascents of this sequence is

$$
\operatorname{asc}\left(x_{1}, \ldots, x_{i}\right)=\left|\left\{1 \leq j<i: x_{j}<x_{j+1}\right\}\right| .
$$

Let us call a sequence $x=\left(x_{1}, \ldots, x_{n}\right) \in \mathbb{N}^{n}$ an ascent sequence of length $n$ if it satisfies $x_{1}=0$ and $x_{i} \in\left[0,1+\operatorname{asc}\left(x_{1}, \ldots, x_{i-1}\right)\right]$ for $2 \leq i \leq n$. For instance, $(0,1,0,2,3,1,0,0,2)$ is an ascent sequence. The length (number of entries) of a sequence $x$ is denoted $|x|$.

Let $\mathcal{S}_{n}$ be the symmetric group on $n$ elements. Let $V=\left\{v_{1}, v_{2}, \ldots, v_{n}\right\}$ with $v_{1}<v_{2}<\cdots<$ $v_{n}$ be any finite subset of $\mathbb{N}$. The standardisation of a permutation $\pi$ on $V$ is the permutation $\operatorname{std}(\pi)$

\footnotetext{
(i) Babson and Steingrímsson call these patterns "generalized" rather than "dashed", but we wish to promote a change of terminology here, since "dashed" is more descriptive.
} 
on $[n]:=\{1,2, \ldots, n\}$ obtained from $\pi$ by replacing the letter $v_{i}$ with the letter $i$. As an example, $\operatorname{std}(19452)=15342$. Let $\mathcal{R}_{n}$ be the following set of permutations:

$$
\mathcal{R}_{n}=\left\{\pi_{1} \ldots \pi_{n} \in \mathcal{S}_{n}: \text { if } \operatorname{std}\left(\pi_{i} \pi_{j} \pi_{k}\right)=231 \text { then } j \neq i+1 \text { or } \pi_{i} \neq \pi_{k}+1\right\} .
$$

Equivalently, if $\pi_{i} \pi_{i+1}$ forms an ascent, then $\pi_{i}-1$ is not found to the right of this ascent. This class of permutations could be more descriptively written as $\mathcal{R}_{n}=\mathcal{S}_{n}\left(\bullet_{\bullet}^{\bullet}\right)$, the set of permutations avoiding the pattern in the diagram. Dark lines indicate adjacent entries (horizontally or vertically) whereas lighter lines indicate an elastic distance between the entries.

As illustrated here, the permutation 31524 avoids the pattern $\because$ - while the permutation 32541 does not.
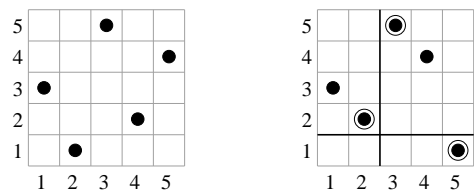

Consider the following three symmetries of a square: reflection in a centered vertical line, reflection in a centered horizontal line, and reflection in the diagonal $x=y$. In the context of permutations these operations are known as reverse, complement and inverse, respectively. Together they generate the dihedral group $D_{8}$, the symmetry group of a square. This is the symmetry of classical patterns. The dashed patterns of Babson and Steingrímsson [1] can be seen as those patterns that allow dark vertical (but not horizontal) lines in their diagram. That set of patterns is not closed under inverse: under reflection in the diagonal $x=y$ a (dark) vertical line turns into a (dark) horizontal line. Thus dashed patterns only enjoy the symmetry of a rectangle. Our patterns provide the minimal extra generality needed to contain the dashed patterns and have the full symmetry of a square.

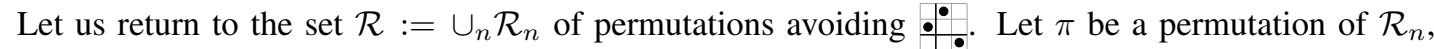
with $n>0$. Let $\tau$ be obtained by deleting the entry $n$ from $\pi$. Then $\tau \in \mathcal{R}_{n-1}$. Indeed, if $\tau_{i} \tau_{i+1} \tau_{j}$ is an occurrence of the forbidden pattern in $\tau$ (but not in $\pi$ ), then this implies that $\pi_{i+1}=n$. But then $\pi_{i} \pi_{i+1} \pi_{j+1}$ would form an occurrence of the forbidden pattern in $\pi$.

This property allows us to construct the permutations of $\mathcal{R}_{n}$ inductively, starting from the empty permutation and adding a new maximal value at each step. Given $\tau=\tau_{1} \ldots \tau_{n-1} \in \mathcal{R}_{n-1}$, the sites where $n$ can be inserted in $\tau$ so as to produce an element of $\mathcal{R}_{n}$ are called active. It is easily seen that the site before $\tau_{1}$ and the site after $\tau_{n-1}$ are always active. The site between the entries $\tau_{i}$ and $\tau_{i+1}$ is active if and only if $\tau_{i}=1$ or $\tau_{i}-1$ is to the left of $\tau_{i}$. Label the active sites, from left to right, with labels $0,1,2 \ldots$

Our bijection $\Lambda$ between permutations of $\mathcal{R}_{n}$ and ascent sequences of length $n$ is defined recursively on $n$ as follows. For $n=1$, we set $\Lambda(1)=(0)$. Now let $n \geq 2$, and suppose that $\pi \in \mathcal{R}_{n}$ is obtained by inserting $n$ in the active site labeled $i$ of a permutation $\tau \in \mathcal{R}_{n-1}$. Then the sequence associated with $\pi$ is $\Lambda(\pi):=\left(x_{1}, \ldots, x_{n-1}, i\right)$, where $\left(x_{1}, \ldots, x_{n-1}\right)=\Lambda(\tau)$.

Example 1 The permutation $\pi=61832547$ corresponds to the sequence $x=(0,1,1,2,2,0,3,1)$, since it is obtained by the following insertions (the subscripts indicate the labels of the active sites):

$$
\begin{aligned}
& { }_{0} 1_{1} \stackrel{x_{2}=1}{\longmapsto} 1_{1} 1_{2} \stackrel{x_{3}=1}{\longmapsto} 0_{0} 1_{1} 3 \quad 2_{2} \stackrel{x_{4}=2}{\longmapsto}{ }_{0} 1_{1} 3 \quad 2_{2} 4_{3} \stackrel{x_{5}=2}{\longmapsto}{ }_{0} 1_{1} 3 \quad 2_{2} 54_{3} \\
& \stackrel{x_{6}=0}{\longmapsto}{ }_{0} 61_{1} 3 \quad 2_{2} 5 \quad 4_{3} \stackrel{x_{7}=3}{\longmapsto}{ }_{0} 61_{1} 32_{2} 54_{3} 7_{4} \stackrel{x_{8}=1}{\longmapsto} \begin{array}{llllllll}
6 & 1 & 8 & 3 & 2 & 5 & 4 & 7 .
\end{array}
\end{aligned}
$$

Theorem 2 The map $\Lambda$ is a bijection from $\mathcal{R}_{n}$ to the set of ascent sequences of length $n$. 
The proof proceeds by induction. The key is to understand how the number of actives sites of $\pi$, and the label located just before its maximal entry, can be read in the ascent sequence.

\section{Ascent sequences and unlabeled $(2+2)$-free posets}

Let $\mathcal{P}_{n}$ be the set of unlabeled $(\mathbf{2}+\mathbf{2})$-free posets on $n$ elements. In this section we shall give a bijection between $\mathcal{P}_{n}$ and the set $\mathcal{A}_{n}$ of ascent sequences of length $n$. As in the previous section, this bijection encodes a recursive way of decomposing $(\mathbf{2}+\mathbf{2})$-free posets by removing one maximal element. This removal procedure is less elementary than in the case of permutations. Before giving these operations we need to introduce some terminology.

Let $D(x)=\{y: y<x\}$ be the set of predecessors of $x$ (the strict down-set of $x$ ). It is well-knownsee for example Khamis [8] - that a poset is $(\mathbf{2}+\mathbf{2})$-free if and only if the set $\{D(x): x \in P\}$ can be linearly ordered by inclusion. Let

$$
D(P)=\left\{D_{0}, D_{1}, \ldots, D_{k}\right\}
$$

with $\emptyset=D_{0} \subset D_{1} \subset \cdots \subset D_{k}$. In this context we define $D_{i}(P)=D_{i}$ and we write $\ell(P)=k$. We say the element $x$ is at level $i$ in $P$ if $D(x)=D_{i}$ and we write $\ell(x)=i$. The set of all elements at level $i$ we denote $L_{i}(P)=\{x \in P: \ell(x)=i\}=\left\{x \in P: D(x)=D_{i}\right\}$. For instance, $L_{0}(P)$ is the set of minimal elements. All the elements of $L_{k}(P)$ are maximal, but there may be maximal elements of $P$ at level less than $k$. If $L_{i}(P)$ contains a maximal element, we say that the level $i$ contains a maximal element. Let $\ell^{\star}(P)$ be the minimum level containing a maximal element.

\section{Example 3}

Consider the following $(\mathbf{2}+\mathbf{2})$-free poset $P$, which we have labeled for convenience. The diagram on the right shows the poset redrawn according to the levels of the elements. We have $D(a)=\{b, c, d, f, g, h\}, D(b)=\emptyset$, $D(c)=D(d)=\{f, g, h\}, D(e)=D(f)=D(g)=$ $\{h\}$ and $D(h)=\emptyset$. These may be ordered by inclusion as
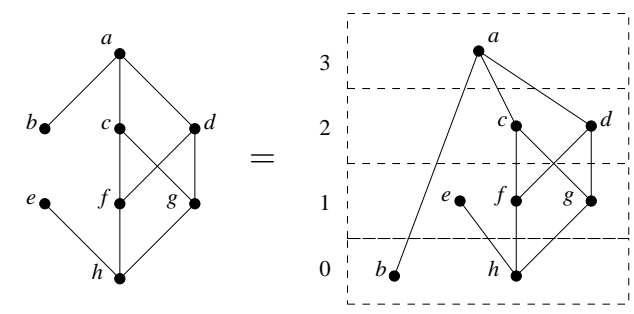

$$
\underbrace{D(h)=D(b)}_{\ell(h)=\ell(b)=0} \subset \underbrace{D(e)=D(f)=D(g)}_{\ell(e)=\ell(f)=\ell(g)=1} \subset \underbrace{D(c)=D(d)}_{\ell(c)=\ell(d)=2} \subset \underbrace{D(a)}_{\ell(a)=3} .
$$

Thus $\ell(P)=3$. The maximal elements of $P$ are $e$ and $a$, and they lie respectively at levels 3 and 1 . Thus $\ell^{\star}(P)=1$. In addition, $D_{0}=\emptyset, D_{1}=\{h\}, D_{2}=\{f, g, h\}$ and $D_{3}=\{b, c, d, f, g, h\}$. With $L_{i}=L_{i}(P)$ we also have $L_{0}=\{h, b\}, L_{1}=\{e, f, g\}, L_{2}=\{c, d\}$ and $L_{3}=\{a\}$.

\subsection{Removing an element from a $(2+2)$-free poset}

The removal operation will be the counterpart of the deletion of the last entry in an ascent sequence (or the deletion of the largest entry in a permutation of $\mathcal{R})$. Let $P$ be a $(\mathbf{2}+\mathbf{2})$-free poset of cardinality $n \geq 2$, and let $i=\ell^{\star}(P)$ be the smallest level of $P$ containing a maximal element. All the maximal elements located at level $i$ are order-equivalent in the unlabeled poset $P$. We will remove one of them. Let $Q$ be the poset that results from applying: 
(Rem1) If $\left|L_{i}(P)\right|>1$ then simply remove one of the maximal elements at level $i$.

(Rem2) If $\left|L_{i}(P)\right|=1$ and $i=\ell(P)$ then remove the unique element lying at level $i$.

(Rem3) If $\left|L_{i}(P)\right|=1$ and $i<\ell(P)$ then set $\mathcal{N}=D_{i+1}(P) \backslash D_{i}(P)$. Make each element of $\mathcal{N}$ a maximal element by deleting from the order all relations $x<y$ where $x \in \mathcal{N}$. Finally, remove the unique element lying at level $i$.

Example 4 Let $P$ be the unlabeled $(2+2)$-free poset with the following Hasse diagram.
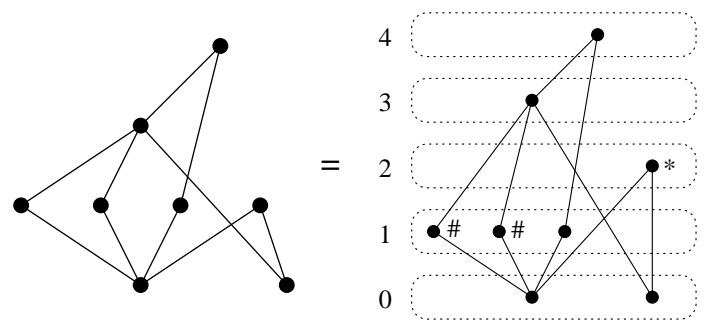

The second diagram shows the poset redrawn according to the levels of the elements. There is a unique maximal element of minimal level, which is marked with $*$, and $\ell^{\star}(P)=2$. Since $2<\ell(P)$, apply Rem3 to remove this maximal element. The elements of $\mathcal{N}$ are indicated by \#'s.

In order to delete all relations of the form $x \leq y$ where $x \in \mathcal{N}$, one deletes all edges corresponding to coverings of elements of $\mathcal{N}$, and adds an edge between the elements at level 0 and 3 to preserve their relation. Finally, one removes the element at level 2. This gives a new $(\mathbf{2}+\mathbf{2})$-free poset, with level numbers shown on the right.
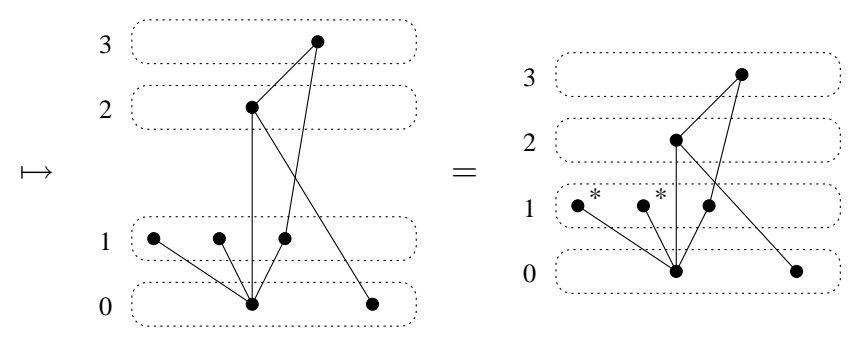

There are now two maximal elements of minimal level $\ell^{\star}=1$, both marked by $*$. Remove one of them according to rule Rem1. This gives the first poset shown to the right, for which $\ell^{\star}$ is still 1. Apply Rem1 again to obtain the second poset on the right.
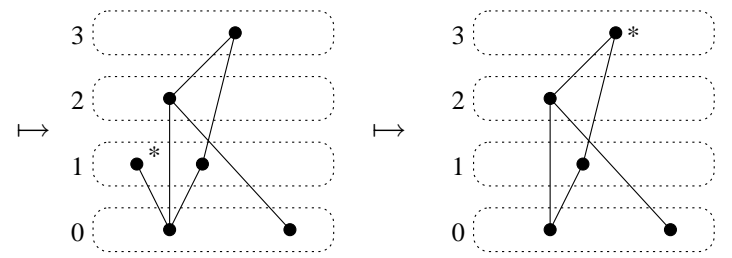

There is now a single maximal element, lying at maximal level 3, so we apply rule Rem2. In the poset thus obtained, $\ell^{\star}(P)=1<$ $\ell(P)$ and there is a unique element at level 1, so apply Rem3. The set $\mathcal{N}$ consists of the rightmost point at level 0 .

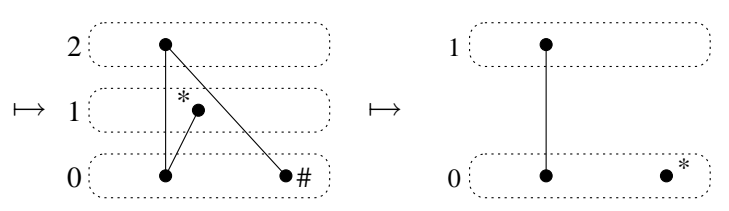

In the new poset, the star element is not alone at level 0, so apply Rem1, and finally Rem2.

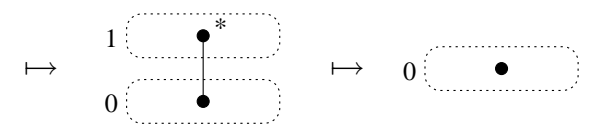

We have thus reduced $P$ to a one element poset by removing the elements in a canonical order. 


\subsection{From $(\mathbf{2}+\mathbf{2})$-free posets to ascent sequences}

Our bijection $\Psi$ between $(\mathbf{2}+\mathbf{2})$-free posets of cardinality $n$ and ascent sequences of length $n$ is defined recursively on $n$ as follows. For $n=1$, we associate with the one-element poset the sequence (0). Now let $n \geq 2$, and suppose that the removal operation, applied to $P \in \mathcal{P}_{n}$, gives the poset $Q$. Then the sequence associated with $P$ is $\Psi(P):=\left(x_{1}, \ldots, x_{n-1}, i\right)$, where $i=\ell^{\star}(P)$ and $\left(x_{1}, \ldots, x_{n-1}\right)=\Psi(Q)$.

For instance, the poset of Example 4 corresponds to the sequence $(0,1,0,1,3,1,1,2)$.

Theorem 5 The map $\Psi$ is a one-to-one correspondence between $(\mathbf{2}+\mathbf{2})$-free posets of size $n$ and ascent sequences of length $n$.

\section{Modified ascent sequences and their applications}

In this section we introduce a transformation on ascent sequences and show some applications. For instance, this transformation can be used to give a non-recursive description of the bijection $\Lambda$ between permutations of $\mathcal{R}$ and ascent sequences. It is also useful to characterize the image by $\Lambda$ of a subclass of $\mathcal{R}$, which we will enumerate in Subsection 4.4. We also describe how to transform $(\mathbf{2}+\mathbf{2})$-free posets into permutations without resorting to ascent sequences.

\subsection{Modified ascent sequences}

Let $x=\left(x_{1}, x_{2}, \ldots, x_{n}\right)$ be any finite sequence of integers. Define

$$
\mathfrak{a s c}(x)=\left(i: i \in[n-1] \text { and } x_{i}<x_{i+1}\right) ;
$$

so $\operatorname{asc}(x)=|\mathfrak{a r c}(x)|$. In terms of an algorithm we shall now describe a function from integer sequences to integer sequences. Let $x=\left(x_{1}, x_{2}, \ldots, x_{n}\right)$ be the input sequence. Do

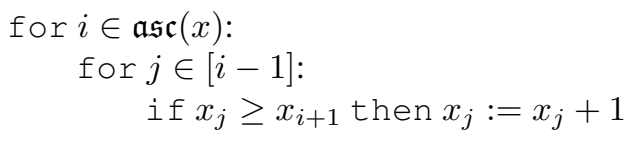

and denote the resulting sequence by $\widehat{x}$. Assuming that $x$ is an ascent sequence we call $\widehat{x}$ the modified ascent sequence. As an example, consider the ascent sequence $x=(0,1,0,1,3,1,1,2)$. We have $\mathfrak{a s c}(x)=(1,3,4,7)$ and the algorithm computes the modified ascent sequence $\widehat{x}$ in the following steps:

$$
x=\begin{array}{llllllll}
0 & 1 & 0 & 1 & 3 & 1 & 1 & 2 \\
0 & 1 & 0 & 1 & 3 & 1 & 1 & 2 \\
0 & 2 & 0 & 1 & 3 & 1 & 1 & 2 \\
0 & 2 & 0 & 1 & 3 & 1 & 1 & 2 \\
0 & 3 & 0 & 1 & 4 & 1 & 1 & 2 \\
& & & & & & &
\end{array}
$$

In each step every element strictly to the left of and weakly larger than the boldface letter is incremented by one. Observe that the positions of ascents in $x$ and $\widehat{x}$ coincide, and that the number of ascents in $x$ (or $\widehat{x}$ ) is $\operatorname{asc}(x)=\operatorname{asc}(\widehat{x})=\max (\widehat{x})$. The above procedure is easily invertible and the map $x \mapsto \widehat{x}$ is therefore injective. 
The modified ascent sequence $\widehat{x}$ is related to the level distribution of the poset $P$ associated with $x$. First, observe that the removal operation of Section 3.1 induces a canonical labelling of the size $n$ poset $P$ by elements of $[n]$ : the first element that is removed gets label $n$, and so on. Applying this to the poset of Example 4 we get the labelling shown on the right.

The following lemma is easily proved by induction.

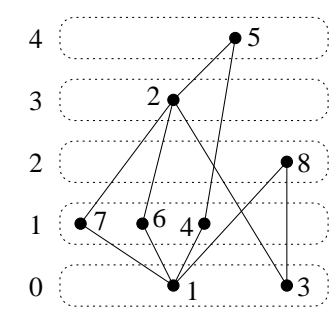

Lemma 6 Let $P$ be a $(\mathbf{2}+\mathbf{2})$-free poset equipped with its canonical labelling. Let $x$ be the associated ascent sequence, and $\widehat{x}=\left(\widehat{x}_{1}, \ldots, \widehat{x}_{n}\right)$ the corresponding modified ascent sequence. Then for all $i \leq n$, the element $i$ of the poset lies at level $\widehat{x}_{i}$.

For instance, listing the elements of the poset above and their respective levels gives

$$
\begin{array}{llllllll}
1 & 2 & 3 & 4 & 5 & 6 & 78 \\
0 & 3 & 0 & 1 & 4 & 1 & 12 \\
0 &
\end{array}
$$

where we recognize the modified ascent sequence of $(0,1,0,1,3,1,1,2)=\Psi(P)$.

\subsection{From posets to permutations}

The canonical labelling of the poset $P$ can also be used to set up the bijection from $(\mathbf{2}+\mathbf{2})$-free posets to permutations of $\mathcal{R}$ without using ascent sequences. We read the elements of the poset by increasing level, and, for a fixed level, in descending order of their labels. This gives a permutation $f(P)$. In our example we get 31764825 , which is the permutation of $\mathcal{R}_{8}$ associated with the ascent sequence $(0,1,0,1,3,1,1,2)=\Psi(P)$.

Proposition 7 For any $(\mathbf{2}+\mathbf{2})$-free poset $P$ equipped with its canonical labelling, the permutation $f(P)$ described above is the permutation of $\mathcal{R}$ corresponding to the ascent sequence $\Psi(P)$. In other words,

$$
\Lambda^{-1} \circ \Psi(P)=\widehat{L}_{0} \widehat{L}_{1} \ldots \widehat{L}_{\ell(P)}:=\pi
$$

where $\widehat{L}_{j}$ is the word obtained by reading the elements of $L_{j}(P)$ is decreasing order. Moreover, the active sites of the above permutation are those preceding and following $\pi$, as well as the sites separating two consecutive factors $\widehat{L}_{j}$.

\subsection{From ascent sequences to permutations, and vice-versa}

By combining Lemma 6 and Proposition 7 we obtain a non-recursive description of the bijection between ascent sequences and permutations of $\mathcal{R}$. Let $x$ be an ascent sequence, and $\widehat{x}$ its modified sequence. Take the sequence $\widehat{x}$ and write the numbers 1 through $n$ below it. In our running example, $x=(0,1,0,1,3,1,1,2)$, this gives

$$
\widehat{x}=\begin{array}{llllllll}
0 & 3 & 0 & 1 & 4 & 1 & 1 & 2 \\
1 & 2 & 3 & 4 & 5 & 6 & 7 & 8
\end{array} .
$$


Let $P$ be the poset associated with $x$. By Lemma 6, the element labeled $i$ in $P$ lies at level $\widehat{x}_{i}$. This information is not sufficient to reconstruct the poset $P$ but it $i$ sufficient to reconstruct the word $f(P)$ obtained by reading the elements of $P$ by increasing level: Sort the pairs $\left(\begin{array}{c}\widehat{x}_{i} \\ i\end{array}\right)$ in ascending order with respect to the top entry and brake ties by sorting in descending order with respect to the bottom entry. In the above example, this gives

\section{$\begin{array}{llllllll}0 & 0 & 1 & 1 & 1 & 2 & 3 & 4\end{array}$}

31764825 .

By Proposition 7, the bottom row, here 31764825 , is the permutation $\Lambda^{-1}(x)$. We have thus established the following direct description of $\Lambda^{-1}$.

Corollary 8 Let $x$ be an ascent sequence. Sorting the pairs $\left(\begin{array}{c}\widehat{x}_{i} \\ i\end{array}\right)$ in the order described above gives the permutation $\pi=\Lambda^{-1}(x)$. Moreover, the number of entries of $\pi$ between the active sites $i$ and $i+1$ is the number of entries of $\widehat{x}$ equal to $i$, for all $i \geq 0$.

The second statement gives a non-recursive way of deriving $x=\Lambda(\pi)$ (or, rather, $\widehat{x}$ ) from $\pi$. Take a permutation $\pi \in \mathcal{R}_{n}$, and indicate its actives sites. For instance, $\pi={ }_{0} 31_{1} 764_{2} 8_{3} 2_{4} 5_{5}$. Write the letter $i$ below all entries $\pi_{j}$ that lie between the active site labeled $i$ and the active site labeled $i+1$ :

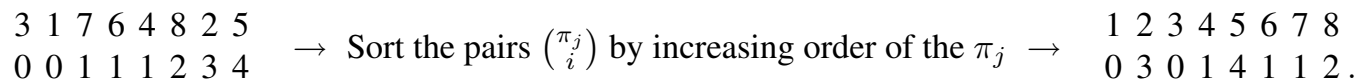

We have recovered, on the bottom row, the modified ascent sequence $\widehat{x}$ corresponding to $\pi$.

\subsection{Permutations avoiding $3 \overline{1} 52 \overline{4}$ and self modified ascent sequences}

A permutation $\pi$ avoids the barred pattern $3 \overline{1} 52 \overline{4}$ if every occurrence of the (classical) pattern 231 plays the role of 352 in an occurrence of the (classical) pattern 31524. In other words, for every $i<j<k$ such that $\pi_{k}<\pi_{i}<\pi_{j}$, there exists $\ell \in(i, j)$ and $m>k$ such that $\pi_{i} \pi_{\ell} \pi_{j} \pi_{k} \pi_{m}$ is an occurrence of 31524 . Note that every such permutation avoids the pattern $\bullet_{-\bullet}^{\bullet}$, and thus belongs to the set $\mathcal{R}$. A conjecture concerning the enumeration of these permutations was given by Pudwell [10, p. 84]. Here, we describe the ascent sequences corresponding to these permutations via the bijection $\Lambda$ from which we can settle her conjecture.

An ascent sequence $x$ is self modified if it is fixed by the map $x \mapsto \widehat{x}$ defined above. For instance, $(0,0,1,0,2,2,0,3,1,1)$ is self modified. In view of the definition of the map $x \mapsto \widehat{x}$, this means that, if $x_{i+1}>x_{i}$, then $x_{j}<x_{i+1}$ for all $j \leq i$.

Proposition 9 The ascent sequence $x$ is self modified if and only if the corresponding permutation $\pi$ avoids $3 \overline{1} 52 \overline{4}$. In this case, $\max (x)=\operatorname{asc}(\pi)=\operatorname{rmin}(\pi)-1$, where $\operatorname{rmin}(\pi)$ is the number of right-toleft minima of $\pi$, that $i$, the number of $i$ such that $\pi_{i}<\pi_{j}$ for all $j>i$.

Recall that $\operatorname{asc}(x)=\max (\widehat{x})$. It is not hard to see that $\left(x_{1}, \ldots, x_{n}\right)$ is a self modified ascent sequence if and only if $x_{1}=0$ and, for all $i \geq 1$, either $x_{i+1} \leq x_{i}$ or $x_{i+1}=1+\max \left\{x_{j}: j \leq i\right\}$. Consequently, a modified ascent sequence $x$ with $\max (x)=k$ reads $0 A_{0} 1 A_{1} 2 A_{2} \ldots k A_{k}$, where $A_{i}$ is a (possibly empty) weakly decreasing factor, and each element of $A_{i}$ is less than or equal to $i$. This structure is the key to count these sequences, and thus permutations avoiding $3 \overline{1} 52 \overline{4}$.

Proposition 10 The length generating function of $3 \overline{1} 52 \overline{4}$-avoiding permutations is $\sum_{k \geq 1} t^{k} /(1-t)\left(\begin{array}{c}k+1 \\ 2\end{array}\right)$. The $k$-th term of this sum counts those permutations that have $k$ right-to-left minima, or, equivalently, $k-1$ ascents. This is also the number of self modified ascent sequences of length $n$ with largest element $k-1$. 


\section{Statistics}

We shall now look at statistics on ascent sequences, permutations and posets—-statistics that we can translate between using our bijections.

Let $x=\left(x_{1}, x_{2}, \ldots, x_{n}\right)$ be any sequence of nonnegative integers. Let last $(x)=x_{n}$. Define zeros $(x)$ as the number of zeros in $x$. A right-to-left maximum of $x$ is a letter with no larger letter to its right; the number of right-to-left maxima is denoted $\operatorname{rmax}(x)$. For example,

$$
\operatorname{rmax}(0,1,0, \mathbf{2}, \mathbf{2}, 0, \mathbf{1})=3 ;
$$

the right-to-left maxima are in bold. For sequences $x$ and $y$ of nonnegative integers, let $x \oplus y=x y^{\prime}$, where $y^{\prime}$ is obtained from $y$ by adding $1+\max (x)$ to each of its letters, and juxtaposition denotes concatenation. For example, $(0,2,0,1) \oplus(0,0)=(0,2,0,1,3,3)$. We say that a sequence $x$ has $k$ components if it is the sum of $k$, but not $k+1$, nonempty nonnegative sequences. Note that $y \oplus z$ is a modified ascent sequence (as defined in Section 4 if and only if $y$ and $z$ are themselves modified ascent sequences. This is the case in the above example.

For any permutation $\pi=\pi_{1} \ldots \pi_{n}$, the statistic $\operatorname{ldr}(\pi)$ (the leftmost decreasing run) is defined as the largest integer $i$ such that $\pi_{1}>\pi_{2}>\cdots>\pi_{i}$. For permutations $\pi$ and $\sigma$, let $\pi \oplus \sigma=\pi \sigma^{\prime}$, where $\sigma^{\prime}$ is obtained from $\sigma$ by adding $|\pi|$ to each of its letters. We say that $\pi$ has $k$ components if it is the sum of $k$, but not $k+1$, nonempty permutations. Observe that $\pi \oplus \sigma$ avoids $\bullet^{\bullet}$ if and only if both $\pi$ and $\sigma$ avoid it. This is the case for instance for $314265=3142 \oplus 21$, which corresponds to the above modified ascent sequence $(0,2,0,1,3,3)=(0,2,0,1) \oplus(0,0)$.

For $\pi \in \mathcal{R}_{n}$, label the active sites with $0,1,2$, etc. Then $b(\pi)$ denotes the label immediately to the left of the maximal entry of $\pi$.

The number of minimal (resp. maximal) elements of a poset $P$ is denoted $\min (P)(\operatorname{resp} . \max (P))$. The ordinal sum of two posets $P$ and $Q$ is the poset $P \oplus Q$ on the union $P \cup Q$ such that $x \leq P \oplus Q y$ if $x \leq_{P} y$, or $x \leq_{Q} y$, or $x \in P$ and $y \in Q$. The definition applies to labeled or unlabeled posets. Let us say that $P$ has $k$ components if it is the ordinal sum of $k$, but not $k+1$, nonempty posets. Observe that $P \oplus Q$ is $(\mathbf{2}+\mathbf{2})$-free if and only if both $P$ and $Q$ are $(\mathbf{2}+\mathbf{2})$-free.

Theorem 11 Given an ascent sequence $x=\left(x_{1}, \ldots, x_{n}\right)$ with modified ascent sequence $\widehat{x}$, let $P$ and $\pi$ be the poset and permutation corresponding to $x$ under the bijections described in Sections 2 and 3 . Then

$$
\begin{aligned}
\left(\min (P), \ell^{\star}(P), \ell(P), \max (P), \operatorname{comp}(P)\right) & =(\operatorname{zeros}(x), \operatorname{last}(x), \operatorname{asc}(x), \operatorname{rmax}(\widehat{x}), \operatorname{comp}(\widehat{x})) \\
& =\left(\operatorname{ldr}(\pi), b(\pi), \operatorname{asc}\left(\pi^{-1}\right), \operatorname{rmax}(\pi), \operatorname{comp}(\pi)\right),
\end{aligned}
$$

where comp denotes the number of components of the individual structures, as defined above.

Example 12 Let $P$ be the poset from Example 4 and let $x$ and $\pi$ be the corresponding ascent sequence and permutation. One checks that the above theorem holds with $\left(\min (P), \ell^{\star}(P), \ell(P), \max (P), \operatorname{comp}(P)\right)=$ $(2,2,4,2,1)$.

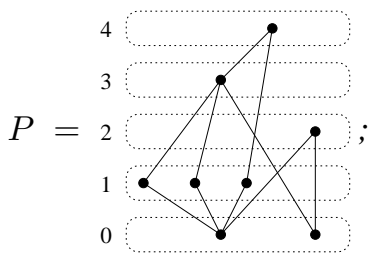

$$
\begin{array}{ll}
x=(0,1,0,1,3,1,1,2) ; & \pi={ }_{0} 31_{1} 764_{2} 8_{3} 2_{4} 5_{5}, \\
\widehat{x}=(0,3,0,1,4,1,1,2) ; & \pi^{-1}=27158436 .
\end{array}
$$




\section{Enumeration}

Theorem 13 Let $p_{n}$ be the number of $(\mathbf{2}+\mathbf{2})$-free posets of cardinality $n$ and let $P(t)=\sum_{n \geq 0} p_{n} t^{n}$ be the associated generating function. Then

$$
P(t)=\sum_{n \geq 0} \prod_{i=1}^{n}\left(1-(1-t)^{i}\right) .
$$

This series also counts permutations of $\mathcal{R}$, and ascent sequences, by length.

To our knowledge, this result is new. El-Zahar [4] and Khamis [8] used a recursive description of $(\mathbf{2}+\mathbf{2})$ free posets, different from that of Section 3 , to derive a pair of functional equations that define the series $P(t)$. However, they did not solve these equations. Haxell, McDonald and Thomasson [7] provided an algorithm, based on a complicated recurrence relation, to produce the first numbers $p_{n}$.

These numbers, and the above expression of $P(t)$, occur in the Encyclopedia of Integer Sequences as sequence A022493 [11]. But there, $P(t)$ is described as counting certain involutions, or chord diagrams [12; 13], that form the topic of Section 7] It is known [13] that

$$
\frac{p_{n}}{n !} \sim \kappa\left(\frac{6}{\pi^{2}}\right)^{n} \sqrt{n}
$$

which proves that the series $P(t)$ is not D-finite (the exponential growth constant would be algebraic).

The proof of Theorem 13 exploits the recursive structure of ascent sequences. The structure translates into a functional equation that defines a 3 -variable generating function $F(t ; u, v)$, which counts these sequences by length $(t)$, ascent number $(u)$ and last entry $(v)$ :

$$
(v-1-t v(1-u)) F(t ; u, v)=(v-1)(1-t u v)-t F(t ; u, 1)+t u v^{2} F(t ; u v, 1) .
$$

The so-called kernel method then gives:

$$
F(t ; u, 1)=\sum_{k \geq 1} \frac{(1-u) u^{k-1}(1-t)^{k}}{\left(u-(u-1)(1-t)^{k}\right) \prod_{i=1}^{k}\left(u-(u-1)(1-t)^{i}\right)} .
$$

Observe that this expression is divergent when $u=1$. In a final step, we transform it into

$$
F(t ; u, 1)=\sum_{n \geq 0} \sum_{\ell=0}^{n}(u-1)^{n-\ell} u^{\ell} \sum_{m=\ell}^{n}(-1)^{n-m}\left(\begin{array}{c}
n \\
m
\end{array}\right)(1-t)^{m-\ell} \prod_{i=m-\ell+1}^{m}\left(1-(1-t)^{i}\right),
$$

which specializes to Theorem 13 when $u=1$.

\section{Involutions with no neighbour nesting}

As discussed above, the series of Theorem 13 is known to count certain involutions on $2 n$ points, called regular linearized chord diagrams (RLCD) by Stoimenow [12]. This result was proved by Zagier [13], following Stoimenow's paper. In this section, we give a new proof of Zagier's result, by constructing a bijection between RLCDs on $2 n$ points and unlabeled $(\mathbf{2}+\mathbf{2})$-free posets of size $n$. 
Let $\mathcal{I}_{2 n}$ be the collection of involutions $\pi$ in $\mathcal{S}_{2 n}$ that have no fixed points and for which every descent crosses the main diagonal in its dot diagram. Equivalently, if $\pi_{i}>\pi_{i+1}$ then $\pi_{i}>i \geq \pi_{i+1}$. An alternative description can be given in terms of the chord diagram of $\pi$, which is obtained by joining the points $i$ and $\pi(i)$ by a chord (Figure 1 left). Indeed, $\pi \in \mathcal{I}_{2 n}$ if and only if, for any $i$, the chords attached to $i$ and $i+1$ are not nested, in the terminology used recently for matchings [3; 9]. That is, the configurations shown on the left of the rules of Fig. 2 are forbidden (but a chord linking $i$ to $i+1$ is allowed).

Recall that a poset $P$ is $(\mathbf{2}+\mathbf{2})$-free if and only if it is an interval order [5]. This means that there exists a collection of intervals on the real line whose relative order is $P$, under the order relation:

$$
[a, b]<[c, d] \Longleftrightarrow b<c .
$$

Let $\pi \in \mathcal{I}_{2 n}$ with transpositions $\left\{\left(\alpha_{i}, \beta_{i}\right)\right\}_{i=1}^{n}$ where $\alpha_{i}<\beta_{i}$ for all $i$. Define $O(\pi)$ to be the interval order (or equivalently, poset) associated with the collection of intervals $\left\{\left[\alpha_{i}, \beta_{i}\right]\right\}_{i=1}^{n}$.

Example 14 Consider $\pi=45712836109 \in \mathcal{I}_{10}$. The transpositions of $\pi$ are shown in the chord diagram of Figure 1 . Beneath the chord diagram is the collection of intervals that corresponds to $\pi$, and the $(\mathbf{2}+\mathbf{2})$-free poset $O(\pi)$ is illustrated on the right hand side. We have added labels to highlight the correspondence between intervals and poset elements.
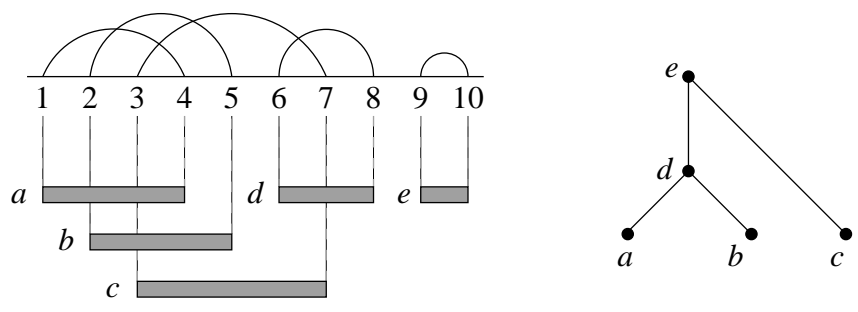

Fig. 1: An involution in $\mathcal{I}_{10}$, the corresponding collection of intervals and the associated $(\mathbf{2}+\mathbf{2})$-free poset.

Theorem 15 The map $O$ is a bijection between involutions of $\mathcal{I}_{2 n}$ and $(\mathbf{2}+\mathbf{2})$-free posets on $n$ elements.

It is not very hard to prove that $O$ is a surjection. That is, for every $(\mathbf{2}+\mathbf{2})$-free order $P$, one can find an involution $\pi$ such that $O(\pi)=P$. The proof uses the transformations of Fig. 2 . We then explain that the involution is uniquely determined by the poset.
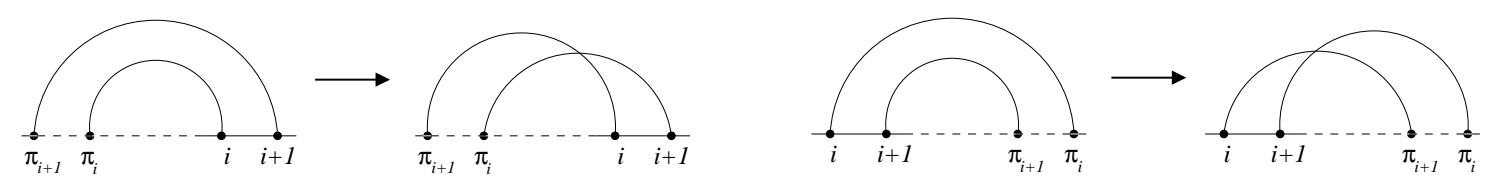

Fig. 2: Two operations on chord diagrams. 


\section{References}

[1] E. Babson and E. Steingrímsson, Generalized permutation patterns and a classification of the Mahonian statistics, Sém. Lothar. Combin. 44 (2000) Art. B44b, 18 pp.

[2] M. Bousquet-Mélou, A. Claesson, M. Dukes and S. Kitaev, Unlabeled $(\mathbf{2}+\mathbf{2})$-free posets, ascent sequences and pattern avoiding permutations, arXiv:0806.0666.

[3] W. Y. C. Chen, E. Y. Deng, R. R. Du, R. P. Stanley, C. H. Yan, Crossings and nestings of matchings and partitions, Trans. Amer. Math. Soc. 359 (2007) 1555-1575.

[4] M. H. El-Zahar, Enumeration of ordered sets, in: I. Rival (Ed.), Algorithms and Order, Kluwer Academic Publishers, Dordrecht, 1989, 327-352.

[5] P. C. Fishburn, Intransitive indifference in preference theory: a survey, Oper. Res. 18 (1970) 207208.

[6] P. C. Fishburn, Intransitive indifference with unequal indifference intervals, J. Math. Psych. 7 (1970) 144-149.

[7] P. E. Haxell, J. J. McDonald and S. K. Thomasson, Counting interval orders, Order 4 (1987) 269272.

[8] S. M. Khamis, Height counting of unlabeled interval and N-free posets, Discrete Math. 275 (2004) 165-175.

[9] C. Krattenthaler, Growth diagrams, and increasing and decreasing chains in fillings of Ferrers shapes, Adv. Appl. Math. 37 (2006), 404-431.

[10] L. Pudwell, Enumeration Schemes for Pattern-Avoiding Words and Permutations, PhD Thesis, Rutgers University, (2008).

[11] N. J. A. Sloane, (2007), The On-Line Encyclopedia of Integer Sequences, published electronically at wWw.research. att. com/ n jas/sequences/.

[12] A. Stoimenow, Enumeration of chord diagrams and an upper bound for Vassiliev invariants, J. Knot Theory Ramifications 7 no. 1 (1998) 93-114.

[13] D. Zagier, Vassiliev invariants and a strange identity related to the Dedeking eta-function, Topology 40 (2001) 945-960. 